\title{
Interprofessional education for collaborative practice: views from a global forum workshop
}

\section{Introduction}

Societal changes around the world have resulted in new public health challenges; larger, more complex and uncoordinated delivery systems encompassing acute and chronic care; a greater reliance on technology; and a serious maldistribution of health workforce resources to address primary care demands. In response, collaboration is becoming an essential component of health promotion and disease management (Humphreys et al., 2012; Leurs, Mur-Veeman, van der Sar, Schaalma, \& de Vries, 2008; Odum \& Whaley-Connell, 2012; Padget, Bekemeier, \& Berkowitz, 2004). The need for effective interprofessional collaboration and teamwork to achieve better health for all and improved personal care at better value requires partnerships beyond the traditional health professions, extending to nonprofessional, frontline health workers, such as community health workers and non-health related professions.

Although there has been growing transformation of delivery systems to address current challenges, educators have been largely unresponsive to the need to transform health professions' education to better align with the needs and expectations of providers and the public. This editorial highlights ideas presented in a summary report of the first two international workshops, hosted by the Institute of Medicine's Global Forum on Innovation in Health Professional Education (IOM, 2013). The report provides descriptions of and reactions to personal experiences with interprofessional collaboration from national and international educators and health providers. Key messages reiterate and extend the substance of the two major reports on which the IOM's Global Forum on Innovation in Health Professional Education is based (Frenk et al., 2010; IOM, 2011).

The Forum report looks at examples of teamwork and collaboration in education and practice that use interprofessional education (IPE) to achieve better patient care; to obtain better health outcomes; and to increase the value of educational and health care systems relative to outcomes. Although we write here as individuals, we were instrumental in planning the workshops and identifying speakers who presented positive patient care and population health outcomes using interprofessional education to enhance the value of interprofessional collaboration.

\section{Global Forum on innovation in health professional education}

The Global Forum is an example of a recent surge in interest in educational redesign. The Forum is composed of 60 members from 17 different professions and eight countries who come together twice a year to network and incubate ideas for innovations in health professional education. It is telling that the first two workshops developed by the Forum were organized around the theme of interprofessional education.

In the US, other indications of the interest in interprofessional education include the formation of the Interprofessional Education Collaborative and the Interprofessional Professionalism Collaborative. The Interprofessional Education Collaborative, a partnership among six US health professions educational associations, has greatly facilitated efforts in interprofessional education by developing a series of core competencies for collaborative practice (IPEC, 2011). These competencies have become a guide for US institutions implementing or refining interprofessional learning activities, and have also become part of the global conversation about competencies for interprofessional collaborative practice. Another US-focused activity, the Interprofessional Professionalism Collaborative, is taking on the specific topic of professionalism across the health professions. And the Josiah Macy Jr. Foundation recently has provided a roadmap for policy changes that would accelerate the linkages between interprofessional education and collaborative practice (Cox \& Naylor, 2013). There is also the recently formed National Center for Interprofessional Practice and Education at the University of Minnesota. It was established with Health Resources and Service Administration funding and the support of four US-based private foundations including Macy, Robert Wood Johnson, Hartford and Gordon, and Betty Moore. The goal of the Center is to coordinate interprofessional efforts between education and practice and it is emerging as a clearinghouse and think tank for interprofessional education and practice. Likewise noteworthy are the three US health professional continuing education accrediting bodies that have been working together since 1998 to develop a joint accreditation process for organizations that support team-based education (ACCME, ACPE, \& ANCC, 2011).

Other important, non-US activities are the Forum-linked projects in Uganda, South Africa, India and Canada. Each of these demonstration projects involves a school of public health, nursing and medicine in the development of an interprofessional curriculum in such areas as leadership and ethics. There are also organized interprofessional networks around the world like the Centre for Advancement of Interprofessional Education, the European Interprofessional Education Network in Health and Social Care, and the Taskforce on Interprofessional Education of The Network: Towards Unity for Health, all of which support the development of interprofessional education. Most of these are represented in the global membership of the 
Forum or were part of the Forum workshop presentations during its first year.

\section{Key workshop issues}

\section{Interprofessional education}

Presenters at the two workshops repeatedly emphasized the importance of interprofessional education, where health professions students learn "'about, from, and with each other', (WHO, 2010). Another key message was that champions of interprofessional education rely heavily upon strong leadership to promote effective learning opportunities. Forum attendees agreed that workplace learning is a critical component of interprofessional education. However, they also noted that many opportunities exist for students from different health professions to learn together in the classroom before beginning clinical training and that these early experiences with interprofessional learning are important as well.

Examples of workplace learning presented at the workshops included a student run clinic for inner-city homeless patients; learning about health disparities from residents of impoverished communities; gaining a better understanding of the challenges faced by outpatients with multiple chronic diseases through a health mentors program; and maximizing simulation technology for learning with and from other professions. The University of Virginia's Collaborative Care Best Practice Model and the Department of Veterans Affairs' Centers of Excellence in Primary Care Education are specific examples of interprofessional learning that span the continuum between pre-clinical education and practice. At Makerere University in Uganda, different health professions are taught together which strengthens their communication skills during their clinical rotations.

For classroom learning, the Medical University of South Carolina has a course on root cause analyses where an interprofessional group of students discuss aspects of a patient's case each from their own professional perspective. This course is part of a university-wide comprehensive, theory-driven approach to interprofessional learning that sets time aside for students of different professions to come together to share their perspectives as a critical component of a truly interprofessional longitudinal curricular experience. Group reflection is fundamental to interprofessional education and establishes a process for lifelong, team-based learning.

\section{Workforce development}

A major challenge for students learning together is that faculty are poorly prepared to teach in an interprofessional environment. Most faculty have not been trained to facilitate or provide feedback to interprofessional teams. Nor are they aware of the enabling interactions and technologies that will assist them in creating a learning community with students and faculty from other health professions. And they have little knowledge of the history, culture and professional development goals of professions other than their own.

Numerous efforts are underway to ensure that the knowledge, skills and behaviors faculty need to implement effective interprofessional education are identified and effectively taught. The Interprofessional Education Collaborative is heavily invested in faculty development. The Josiah Macy Jr. Foundation is supporting professional development activities. Many university and health system endeavors are focused on teamwork and patient safety. Continuing education efforts are also beginning to make interprofessional learning goals more explicit. Other efforts focus on building a more diverse faculty, and include not only traditional clinical educators but role models in the practice environment as well. Strengthening linkages between educators and practitioners who model interprofessional care ensures that messages from the explicit and from the "hidden" curriculum are consistent throughout the student's learning experience.

Although the best way to harmonize formal teaching with experience is to expose students only to well-functioning interprofessional teams, this is not always possible. Educators at the University of Colorado and Curtin University in Australia are using a "hub and spoke" model in which students are first exposed to a range of interprofessional experiences then return to their classroom for an interprofessional, facilitated discussion on the differences in the quality of team functioning experienced.

\section{Patient, family and community involvement}

An important point expressed at the workshop by both members and patient advocates was the urgency of including patients, families and caretakers as central members of health care teams and the importance of involving communities in the design of interventions to meet population health needs. This requires the academic institutions to be engage in a direct interaction with community stakeholders and demonstrate their "social accountability" by being responsive to community needs. The medical school at Ghent University in Belgium is one such example. For the last decade, a one-week course has been offered at the school that partners medical students with master of social work and social welfare studies students to jointly undertake a community diagnosis exercise (Art, De Roo, Willems, \& De Maeseneer, 2008). As part of the exercise, small groups of students visit patients and their caregivers living in underserved urban neighborhoods. This home-visit experience is then combined with public health data in order to build a community diagnosis. Such experiential learning is valuable to students and contributes to the social accountability of the university. Two other examples of social accountability that are noted in the report came from Duke University in North Carolina and Flinders University in Australia. At Duke, faculty and practitioners are weaving health into various aspects of the communities they serve largely through affiliations with health departments; and at Flinders, university leaders are focusing on improving health in rural communities by building the quality and quantity of health providers in remote parts of Australia.

\section{Measuring the value of interprofessional education}

Numerous examples showing benefits of student exposure to well-functioning teams are noted in the workshop summary report. A leader in this area is Kaiser Permanente's Department of Pharmacy in the Colorado Region. Their program places students in 27 different interprofessional practice sites that benefit from the student placement, while the student learns about collaboration from highly functioning teams. It is also evident that students crave a more interprofessional approach to learning. Moreover, employers such as IBM, the Veteran's Health Administration, and Kaiser Permanente and national health systems like the National Health Service in the United Kingdom are increasingly concerned that present graduates do not possess the skills needed to work in a collaborative environment. If clinical workforce needs alone are an insufficient reason, students' interest and the demands of large employers may force the academic community to reconsider its present reluctance to invest in interprofessional education.

Despite the steady accumulation of information promoting the value of interprofessional education in building stronger ties among professionals, patients and communities, there is a lack of robust evidence confirming that it improves patient outcomes. 
impacts population health, or tempers the rise in health care costs (Reeves, Perrier, Goldman, Freeth, \& Zwarenstein, 2013). Many believe this standard is uniquely set for interprofessional education and collaborative practice and that traditional forms of health professional education are not similarly challenged. Be this as it may, researchers are now setting forth to test the value of interprofessional training through well-designed studies.

\section{Concluding comments}

Movements toward more effective teamwork and stronger collaborations are underway although the problem of professional silos remains in both educational and care settings. Interprofessional education can be an important mechanism for overcoming such barriers. Training students to become "change agents" who continuously improve the health care process will contribute to this aim. With all the efforts underway to promote interprofessional education, there is no doubt that momentum will continue to grow. More and more students and health professional leaders will come to understand the importance of collaboration, and patients and the public will increasingly demand a more comprehensive approach and improved communication among their health professionals. All this starts with interprofessional education. Interprofessional education is a tool for changing the way students are educated and the way health professionals work together, mentor students, and learn with and from patients and the communities they serve.

Patricia Cuff

Forum Director and Senior Program Officer Board on Global Health Institute of Medicine, Washington, DC, USA E-mail:PCuff@nas.edu

Madeline Schmitt

University of Rochester, Rochester, USA

Brenda Zierler

University of Washington, Seattle, USA

Malcolm Cox

U.S. Department of Veterans Affairs, Washington DC, USA

Jan De Maeseneer Ghent University, Ghent, Belgium

Lucinda L. Maine American Association of Colleges of Pharmacy, Washington DC, USA

Scott Reeves

University of California, San Francisco, San Francisco, USA

Harrison C. Spencer Association of Schools of Public Health, Washington DC, USA

George E. Thibault

Josiah Macy, Jr. Foundation, New York, USA

\section{Declaration of interest}

The authors are responsible for the content of this article, which does not necessarily represent the views of the Institute of Medicine. The authors declare no conflicting interests. The authors are responsible for the writing and content of this editorial.

\section{References}

ACCME (Accreditation Council for Continuing Medical Education), ACPE (Accreditation Council for Pharmacy Education) and ANCC (American Nurses Credentialing Center). (2011). Joint accreditation eligibility, criteria and policies. Available at: http://www.accme.org/ sites/default/files/null/Joint_Accred_CE_Healthcare_Team_Packet_20 111023.pdf

Art, B., De Roo, L., Willems, S., \& De Maeseneer, J. (2008). An interdisciplinary community diagnosis experience in an undergraduate medical curriculum: Development at Ghent University. Academic Medicine, 83, 675-683.

Cox, M., \& Naylor, M. (2013). Transforming patient care: Aligning interprofessional education with clinical practice redesign. Proceedings of a Conference sponsored by the Josiah Macy Jr. Foundation in January 2013; New York: Josiah Macy Jr. Foundation.

Frenk, J., Chen, L., Bhutta, Z.A., Cohen, J., Crisp, N., Evans, T., Fineberg, H., et al. (2010). Health professionals for a new century: Transforming education to strengthen health systems in an interdependent world. The Lancet, 376, 1923-1958.

Humphreys, J., Harvey, G., Coleiro, M., Butler, B., Barclay, A., Gwozdziewicz, M., O’Donoghue, D., \& Hegarty, J. (2012). A collaborative project to improve identification and management of patients with chronic kidney disease in a primary care setting in Greater Manchester. BMJ Quality and Safety, 21, 700-708.

IOM (Institute of Medicine). (2011). The future of nursing: Leading change, advancing health. Washington, DC: The National Academies Press.

IOM (Institute of Medicine). (2013). Interprofessional education for collaboration: Learning how to improve health from interprofessional models across the continuum of education to practice: Workshop summary. Washington, DC: The National Academies Press.

IPEC (Interprofessional Education Collaborative Expert Panel). (2011). Core competencies for interprofessional collaborative practice: Report of an expert panel. Washington, DC: Interprofessional Education Collaborative.

Leurs, M., Mur-Veeman, I., van der Sar, R., Schaalma, H., \& de Vries, N. (2008). Diagnosis of sustainable collaboration in health promotion - a case study. BMC Public Health, 8, 382.

Odum, L., \& Whaley-Connell, A. (2012). The role of team-based care involving pharmacists to improve cardiovascular and renal outcomes. Cardiorenal Medicine, 2, 243-250.

Padget, S., Bekemeier, B., \& Berkowitz, B. (2004). Collaborative partnerships at the state level: Promoting systems changes in public health infrastructure. Journal of Public Health Management Practice, 10, 251-257.

Reeves, S., Perrier, L., Goldman, J., Freeth, D., \& Zwarenstein, M. (2013). Interprofessional education: Effects on professional practice and healthcare outcomes (update). Cochrane Database of Systematic Reviews, Issue 3.

WHO (World Health Organization). (2010). Framework for action on interprofessional education \& collaborative practice. Geneva, Switzerland: World Health Organization, Department of Human Resources for Health. 